\title{
Dentin matrix protein 1 and phosphate homeostasis are critical for postnatal pulp, dentin and enamel formation
}

\author{
Afsaneh Rangiani, Zheng-Guo Cao, Ying Liu, Anika Voisey Rodgers, Yong Jiang, Chun-Lin Qin \\ and Jian-Quan Feng
}

Deletion or mutation of dentin matrix protein 1 (DMP1) leads to hypophosphatemic rickets and defects within the dentin. However, it is largely unknown if this pathological change is a direct role of DMP1 or an indirect role of phosphate (Pi) or both. It has also been previously shown that Klotho-deficient mice, which displayed a high Pi level due to a failure of Pi excretion, causes mild defects in the dentinal structure. This study was to address the distinct roles of DMP1 and Pi homeostasis in cell differentiation, apoptosis and mineralization of dentin and enamel. Our working hypothesis was that a stable Pi homeostasis is critical for postnatal tooth formation, and that DMP1 has an antiapoptotic role in both amelogenesis and dentinogenesis. To test this hypothesis, Dmp1-null $\left(D m p 1^{-/}\right)$, Klotho-deficient ( $k l / k n), D m p 1 / K l o t h o-d o u b l e-d e f i c i e n t ~\left(D m p 1^{-1-} / k l / k I\right)$ and wild-type (WT) mice were killed at the age of 6 weeks. Combinations of X-ray, microcomputed tomography $(\mu \mathrm{CT})$, scanning electron microscopy (SEM), histology, apoptosis and immunohistochemical methods were used for characterization of dentin, enamel and pulp structures in these mutant mice. Our results showed that $\mathrm{Dmp1}^{-/-}$(a low Pi level) or $\mathrm{kl} / \mathrm{kl}$ (a high Pi level) mice displayed mild dentin defects such as thin dentin and a reduction of dentin tubules. Neither deficient mouse line exhibited any apparent changes in enamel or pulp structure. However, the double-deficient mice (a high Pi level) displayed severe defects in dentin and enamel structures, including loss of dentinal tubules and enamel prisms, as well as unexpected ectopic ossification within the pulp root canal. TUNEL assay showed a sharp increase in apoptotic cells in ameloblasts and odontoblasts. Based on the above findings, we conclude that DMP1 has a protective role for odontoblasts and ameloblasts in a pro-apoptotic environment (a high Pi level).

International Journal of Oral Science (2012) 4, 189-195; doi:10.1038/ijos.2012.69; published online 21 December 2012

Keywords: apoptosis; dentin; dentin matrix protein 1; enamel; Klotho; phosphate

\section{INTRODUCTION}

Dentin matrix protein 1 (DMP1), an extracellular matrix phosphoprotein belonging to small integrin ligand N-linked glycoprotein family, ${ }^{1}$ plays multiple functions in mineralized tissues. ${ }^{2}$ One of the major disturbances observed in Dmp1-null animals is the defective dentin formation and mineralization, including a reduced dentin apposition rate, increased predentin width, and a disorganized dentinal tubule arrangement, ${ }^{3-4}$ as well as changes of phosphate (Pi) homeostasis caused by abnormal increases of fibroblast growth factor (FGF) $23 .{ }^{5}$ Mutations in human DMP1 cause autosomal recessive hypophosphatemic rickets. ${ }^{5-6}$ One human Dmp1 deletion mutation case had a deletion of nucleotides 1484 to 1490 (c.1484_1490delCTATCAC) resulting in replacement of the last 18 residues with 33 random amino acids, displayed severe dentin and enamel defects similar to a dentinogenesis imperfecta III-like phenotype. ${ }^{7}$ Our previous studies suggest that the C-terminal of DMP1 is likely a key functional domain of this matrix protein. ${ }^{7-8}$ Currently, the mechanisms for these abnormal changes are not clear, although the defects are most likely attributed to a combination of the local role of DMP1 and a change of the systemic phosphate homeostasis. ${ }^{9}$

Klotho, a type I transmembrane protein, is an obligatory co-receptor for FGF23. ${ }^{10}$ In FGF23 mediated signaling, FGF receptors are able to bind FGF23 with high affinity only in the presence of Klotho. ${ }^{11}$ FGFRs are ubiquitously expressed in different tissues but Klotho is only expressed in limited tissues such as kidney, parathyroid glands and brain, which gives FGF23's function tissue specificity. ${ }^{12}$ Klothonull mice show aging phenotypes such as hair loss, infertility and emphysema. Within these mice, there is a dramatic increase in the serum levels of phosphate, calcium, vitamin D and FGF23. Hyperphosphatemia is the result of impaired FGF23 signaling in the kidney, regarding the role of Klotho in converting FGFR1 to FGF23 receptor. ${ }^{113}$ Both Fgf23-null and Klotho-deficient mice are reported to have mild dentin malformation and increased apoptosis in odontoblasts, which is due to a high phosphate level. ${ }^{14-15}$

A key regulator of FGF23 is DMP1, ${ }^{16-17}$ which is also detected transiently in developing ameloblasts, but it is not detected in mature enamel. ${ }^{18}$ In our previous studies, ${ }^{19}$ we showed that $D m p 1^{-/-} /$Klotho $\left(D m p 1^{-/-} / k l / k l\right)$ double-deficient mice were hyperphosphatemic. This hyperphosphatemia resulted in increased apoptosis and ectopic mineralization in cells expressing DMP1, including osteocytes, endothelial cells and renal tubule cells within the kidney. ${ }^{19}$

In this study, we sought to determine the distinct roles of DMP1 and Pi homeostasis in cell differentiation, apoptosis and mineralization of 
dentin and enamel. Our findings suggest that: (i) DMP1 has an important local role in dentinogenesis, amelogenesis and dentinal tubule formation; and (ii) within the ameloblasts and odontoblasts, DMP1 has a protective role against apoptotic effects of hyperphosphatemia secondary to Klotho deficiency (note that Klotho is not expressed in either of these cells). These findings may facilitate future drug development in conditions with increased phosphate levels such as chronic kidney disease or tumoral calcinosis for the toxic effect of hyperphosphatemia.

\section{MATERIAL AND METHODS}

Animals

Klotho-deficient mice $(k l / k l)$ and Dmpl lacZ knock-in-null mice $\left(D m p 1^{-1-}\right.$ ) were described previously. ${ }^{20-21}$ The $k l / k l$ mouse (provided kindly by Dr Kuro-o's lab) was originally described as a severe hypomorph strain, because extremely low levels of Klotho mRNA were detectable only by reverse transcription polymerase chain reaction (PCR) ${ }^{20}$ However, Klotho protein was undetectable in any tissues by immunoblot, immunoprecipitation and immunohisotchemical analyses (data not shown). In addition, $\mathrm{kl} / \mathrm{kl}$ mice were shown to exhibit the same phenotypes as Klotho-deficient mice. ${ }^{22}$ Thus, the $k l / k l$ mouse is virtually equivalent to a null strain. All the mice were fed with tap water and autoclaved Purina rodent chow (5010, Rastlon Purina) containing calcium, $0.67 \%$ phosphorus and 4.4 international units of vitamin D per gram.

\section{Generation of $D m p 1^{-/-} / k l / k l$ compound-deficient mice}

Dmp1 and Klotho murine genes are located on chromosome 5 (qE5 and qG3 respectively). The generation of Dmp1/Klotho compound deficient mice $\left(D m p 1^{-/-} / k l / k l\right)$ was based on the chance of crossover between homologous chromosomes. First, we crossed $D m p 1^{-1-}$ and Klotho-deficient heterozygous mice $(k l /+)$. Their offspring will include male and female heterozygous mice for both Dmpl and Klotho $\left(D m p 1^{+/-} / k l /+\right)$ which were set for litter-mating. In the first three generations, no cross-over occurred. After the third generation, Dmp1-null Klotho hetero animals $\left(\mathrm{Dmpl}^{-/-} / \mathrm{kl} /+\right)$ were born and used for mating with a one-in-four chance for producing compound homozygous mice $\left(D m p 1^{-/-} / k l / k l\right)$. At the same time, compound hetero mice $\left(D m p 1^{+/-} / k l /+\right)$ were also continued mating to generate wildtype (WT), Dmp1 ${ }^{-/-}$and $k l / k l$ animals; A few $D m p 1^{-/-} / k l / k l$ mice were also born through this set of mating.

\section{Genotyping}

DNA was extracted from the toe of each mouse by standard protocol and subjected to PCR for genotyping. $D m p 1^{+/-}$and $D m p 1^{-/-}$were genotyped by PCR as reported previously. ${ }^{21}$ For $D m p 1$, the PCR program consisted of $4 \mathrm{~min}$ initial denaturation at $94{ }^{\circ} \mathrm{C}$, amplification cycle including denaturation at $94{ }^{\circ} \mathrm{C}$ for $1 \mathrm{~min}$, annealing at $55{ }^{\circ} \mathrm{C}$ for $2 \mathrm{~min}$, and extension for $3 \mathrm{~min}$ at $72{ }^{\circ} \mathrm{C}$, and final extension was performed at $72{ }^{\circ} \mathrm{C}$ for $10 \mathrm{~min}$. The expected product size for the targeted Dmp1 allele was $280 \mathrm{bp}$ and the WT allele was $410 \mathrm{bp}$. The Klotho genotyping protocol and the primers used have been previously mentioned. ${ }^{23}$ Briefly for Klotho genotyping, Takara LA Taq and buffer were used and the protocol for PCR included 2 min initial denaturation at $94{ }^{\circ} \mathrm{C}$, amplification cycle consisting denaturation at $94{ }^{\circ} \mathrm{C}$ for $30 \mathrm{~s}$, annealing at $56{ }^{\circ} \mathrm{C}$ for $30 \mathrm{~s}$, and extension for $1.5 \mathrm{~min}$ at $72{ }^{\circ} \mathrm{C}$, which was followed by final extension at $72{ }^{\circ} \mathrm{C}$ for $10 \mathrm{~min}$. The expected product size for WT Klotho was $458 \mathrm{bp}$ and for mutant Klotho was 920 bp.

\section{Histology, immunohistochemistry and TUNEL staining}

The mandibles of 6-week-old mice were collected, fixed in $4 \%$ paraformaldehyde in phosphate buffered saline ( $\mathrm{pH} 7.4)$ for $48 \mathrm{~h}$, decalcified by microwave ethylene diaminetetraacetic acid (EDTA) for $16 \mathrm{~h}$, dehydrated through graded alcohol and then embedded in paraffin. Sections were cut $4.5 \mu \mathrm{m}$ thick and were mounted on slides and dried. Slides of mandibular incisor and first molar were used for regular hematoxylin and eosin (H\&E) staining. For immunohistochemistry, sclerostin (SOST), nestin, dentin sialophosphoprotein (DSPP) antibodies were used on mandibular first molars of four study groups including WT, $D m p 1^{-/-}, k l / k l$ and $D m p 1^{-/-} / \mathrm{kl} / \mathrm{kl}$ animals. To analyze apoptosis in enamel and dentin tissues of the mandibular incisor, the TUNEL kit (Roche Diagnostics, Indianapolis, IN, USA) was used.

\section{Microcomputed tomography, radiography and scanning electron microscopy}

After the fixation process, mandibles were dissected and radiographs were taken using Faxitron radiographic inspection unit (model 8050; Field Emission Corporation, Inc., McMinnville, OR, USA), with digital capture image capability.

Mandibular incisoral enamel and dentin compartments were contoured out and imaged using an X-ray microCT imaging system ( $\mu$ CT 35, Scanco Medical, Basserdorf, Switzerland). Serial tomographic imaging at an energy level of $55 \mathrm{kV}$ and intensity of $145 \mu \mathrm{A}$ for the mandibles was performed.

To image the odontoblast tubular system, scanning electron microscopy (SEM) of resin casted bone samples was performed. Mandibles were fixed in $70 \%$ ethanol and embedded in methyl methacrylate (Buehler, Lake Bluff, IL, USA). The surface of the methyl methacrylate-embedded bone was polished, followed by acid etching with $37 \%$ phosphoric acid for 2-10 s, 5\% sodium hypochlorite for $5 \mathrm{~min}$, and coating with gold and palladium. Samples were examined by an FEI/ Philips XL30 field emission environmental SEM (Phillips, Hillsboro, OR, USA). For backscattered electron microscopy imaging, we used the method described previously. ${ }^{24}$

\section{RESULTS}

Compound $D m p 1^{-/-} / k l / k l$-deficient mice displayed severe enamel defects in the incisoral proximal region

To address whether a potential effect of a low Pi level $\left(D m p 1^{-/-}\right)$or a high Pi level $(k l / k l)$ or a loss of both genes (the compound mice displaying a high $\mathrm{Pi}$ level) on enamel we first showed that there were no apparent changes in molar enamel in all groups examined with microcomputed tomograph $(\mu \mathrm{CT})$ imaging. Unexpectedly, the compound-deficient mice showed a loss of more than $95 \%$ of mineral in the proximal incisoral enamel region, which was formed after birth (Figure la, right image, red arrow), whereas there was no apparent change in the distal enamel compared to the WT control. Next, we observed irregular enamel prism structure along with disordered enamel rod arrangement within in this area using SEM (Figure 1b, red arrow), although there was no change in the compound-deficient molar enamel structure (Figure 1c). These pathological changes in the proximal enamel region (reflecting postnatal developmental stage), but not in the distal region or molars (reflecting early developmental stage) suggesting that the defect within the formation, maturation and mineralization of enamel is caused by a combination of a loss of DMP1 local function and a high Pi environment in the system.

\section{Compound $D m p 1^{-/-} / k l / k l$-deficient mice exhibited ectopic} calcification in the root dentin

Previously, we reported dentin defects in $D m p 1^{-1-}$ mice. ${ }^{3}$ Here we showed a thin dentin phenotype in the $D m p 1^{-/-}$mice compared to 

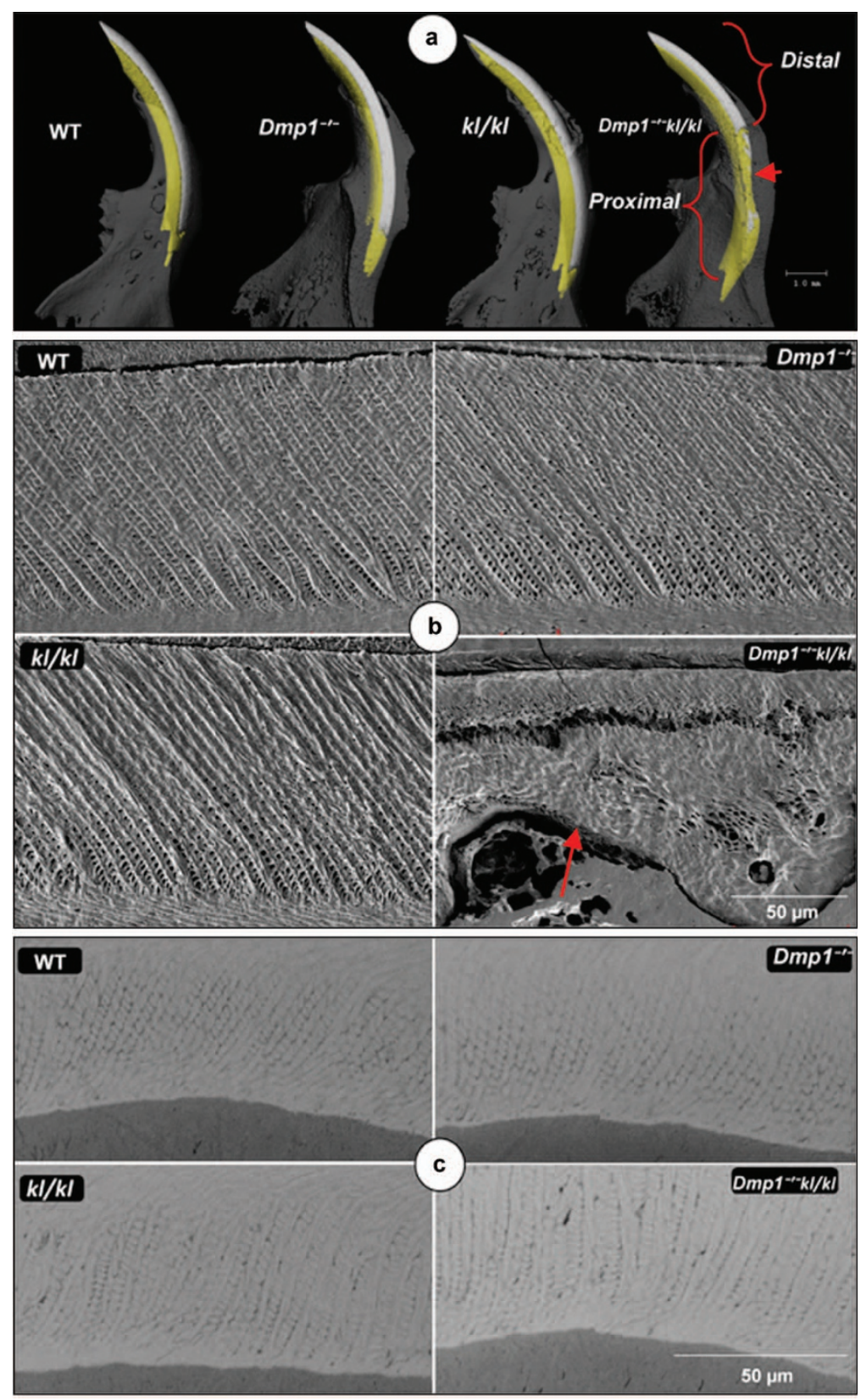

Figure 1 Deletion of Dmp 1 in a high phosphate background resulted in severe enamel malformation. (a) Representative $\mu$ CT images showed a lack of enamel in the proximal region of the lower incisor obtained from the Dmp1/Klotho-double-deficient mice (red arrow); (b) acid etching resin-casted SEM images revealed malformed enamel prisms in the proximal region of the compound-deficient mice (red arrow); and (c) backscattered SEM images displayed no apparent difference in the first molar among four groups. SEM, scanning electron microscopy; $\mu \mathrm{CT}$, microcomputed tomography.

the WT control by a $\mu \mathrm{CT}$ approach (Figure 2a, white arrow) and no obvious changes were evident in the overall dentin structure in the $k l / k l$ mice. Interestingly, the $D m p 1^{-/-} / \mathrm{kl} / \mathrm{kl}$ mice exhibited an extremely thin dentin with ectopic calcified structures in the root canal (Figure 2a, right image, yellow arrows). The H\&E image revealed a totally different morphological structure from the surrounding dentin structure: numerous cementocyte- or osteocyte-like cells within the ectopic tissue (Figure 2b, right image, yellow arrows). The backscattered SEM image further confirmed a cementocyte- or osteocyte-like cell with a completely different matrix from a normal dentin structure (Figure 2c).

To understand the nature of the ectopic calcified structure we tested the following markers: bone sialoprotein (a marker for odontoblasts or osteoblasts but not for osteocytes), DSPP (a marker mainly for dentin), nestin (a marker for odontoblasts but not for bone cells) and SOST (a marker for osteocytes only). As shown in Figure 3, bone sialoprotein and DSPP were detected in the ectopic tissue with a low expression level, although neither nestin nor SOST was observed in the ectopic tissue. These results indicate that the ectopic calcified tissues do not fit as a dentin, or a bone, or a cementum structure.

\section{Compound $D m p 1^{-/-} / k l / k l$-deficient mice displayed severe dentinal} structure defects

In search for potential changes in the dentinal structure in these mouse models, we initially showed a reduction in dentin tubule numbers and 

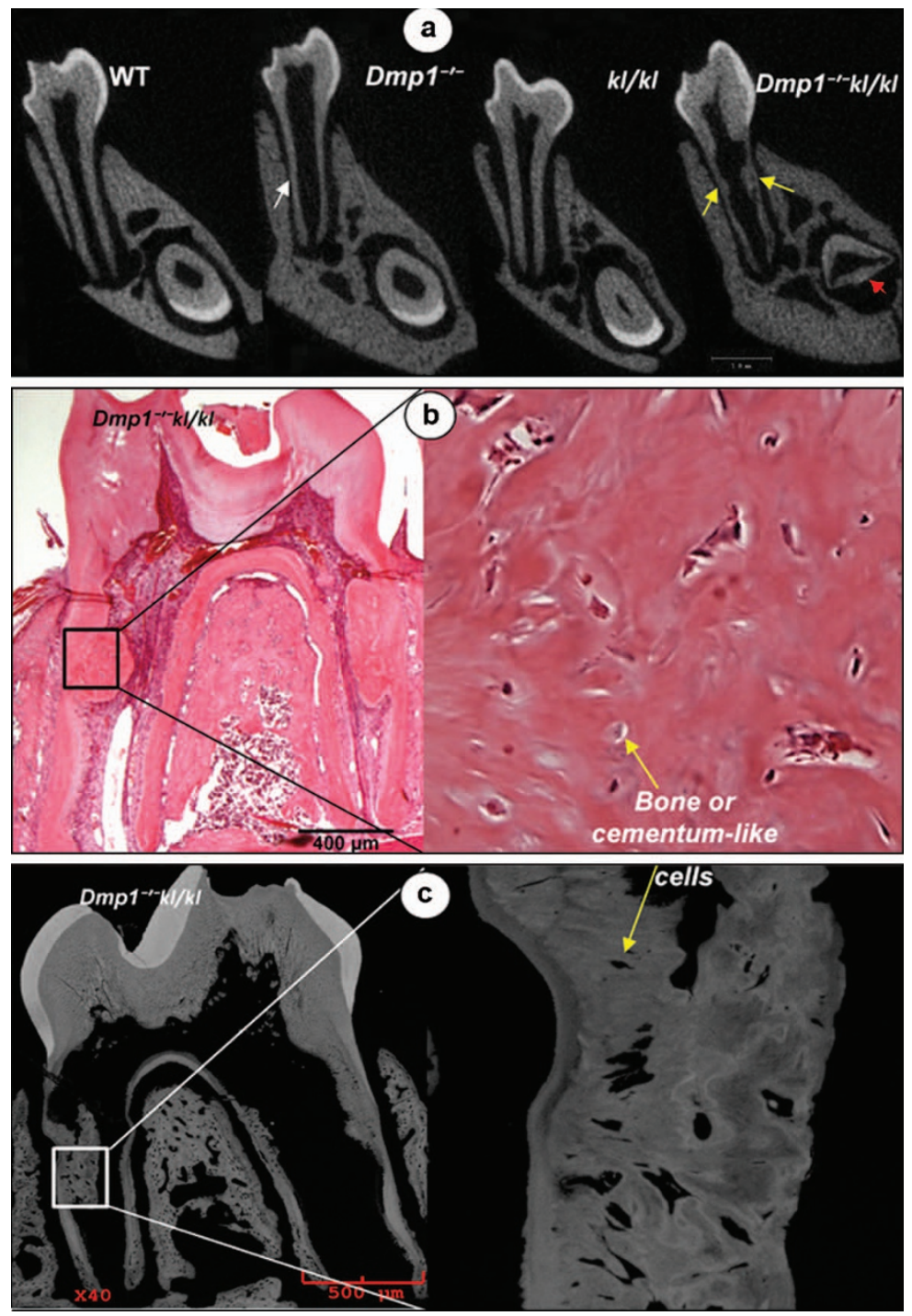

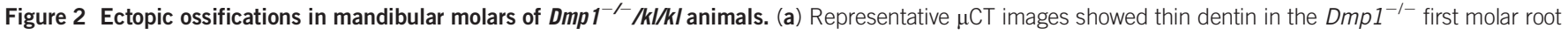
(white arrow), no apparent change in overall tooth structure in the $\mathrm{kl} / \mathrm{k} / \mathrm{mouse}$, and ectopic ossifications in the compound deficient mice first molar root (yellow arrows) and malformed incisor (red arrow); (b) H\&E images exhibited bone or cementum-like cells in the ectopic tissue with a totally different matrix from a regular dentin matrix in the compound deficient first molar; and (c) backscattered SEM images confirmed the malformed ossified structure. H\&E, hematoxylin and eosin; SEM, scanning electron microscopy; $\mu \mathrm{CT}$, microcomputed tomography.
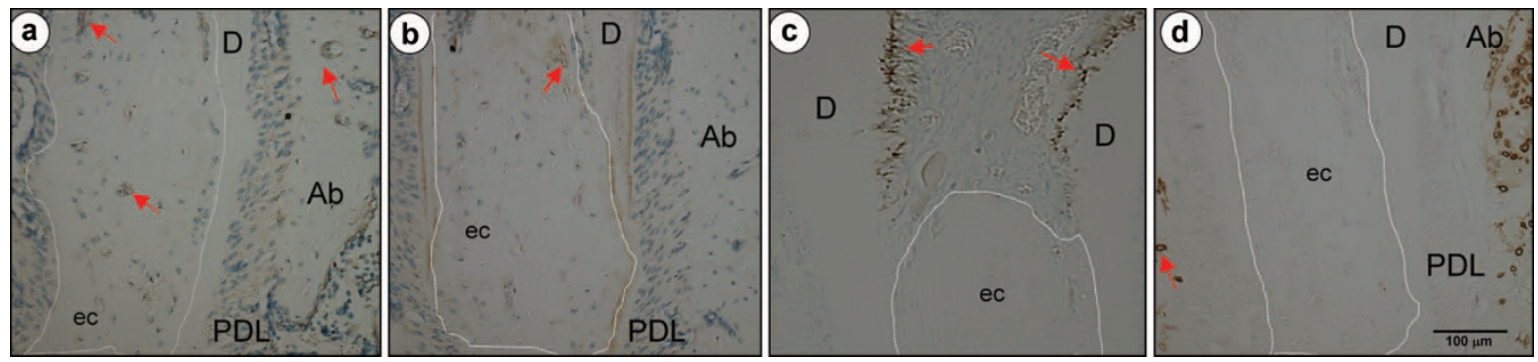

Figure $3 \mathrm{IHC}$ profiles of the ectopic ossification tissues obtained from the mandibular first molar of $\mathbf{D m p} \mathbf{1}^{-/} / \mathbf{k l} / \mathbf{k} \mathbf{l}$ mouse. (a) BSP expression profile showing a similar intensity as that in Ab; (b) DSPP expression profile displaying signal in patch in contrast to the smooth signal in dentin; (c) nestin expression profile revealing no signal in the ectopic ossified region, whereas a strong signal shown in the odontoblast layer (red arrows); and (d) SOST expression profile showed no signal in the ectopic ossified tissue but a strong signal in alveolar bone. Ab, alveolar bone; BSP, bone sialoprotein; DSPP, dentin sialophosphoprotein; IHC, immunohistochemistry; SOST, sclerostin. 

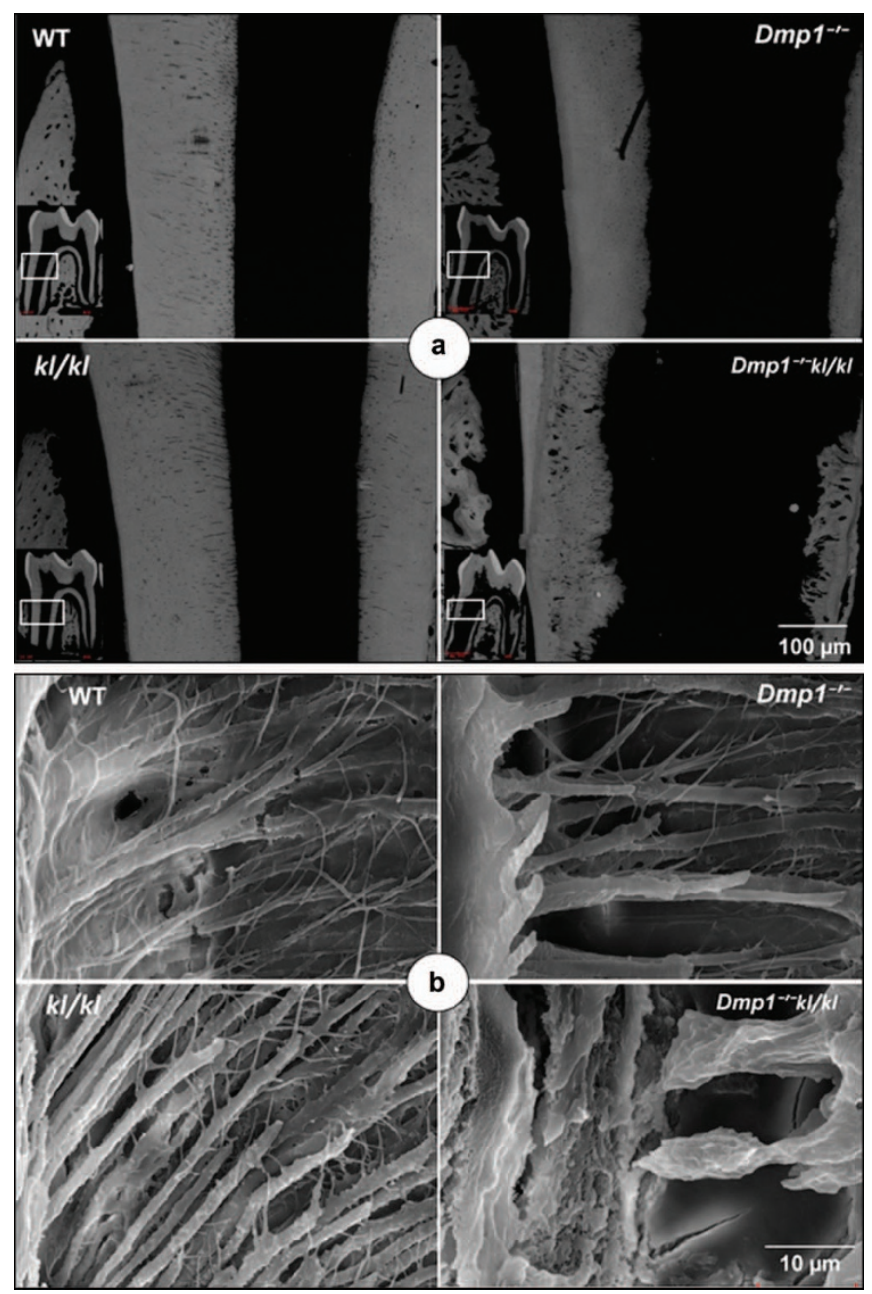

Figure 4 Severe defects in dentin size and dentin tubule in the compound deficient mice. (a) Representative backscattered SEM images showed relatively thin root dentin in the $\mathrm{Dmp1}^{-1-}$ mouse (upper right image), no apparent change in overall root and dentin structure in $\mathrm{kl} / \mathrm{k} / \mathrm{mice}$, and extremely thin dentin without 'holes' (reflecting dentin tubules) plus ectopic ossification structure (displaying cell-like structure similar to osteocytes or cementocytes); (b) acid etching resincasted SEM images revealed minor changes in $\mathrm{Dmp1}^{-/-}$dentin tubules (upper right image), little changes in $\mathrm{kl} / \mathrm{k} /$ dentin tubules (lower left image); and a loss of dentin feature in the compound deficient mice (lower right image). SEM, scanning electron microscopy.

thickness in the $D m p 1^{-1-}$ molars by using both backscattered SEM (Figure 4a, right upper image) and acid etching SEM approaches (Figure $4 \mathrm{~b}$, right upper image), which is in agreement with our previous report. ${ }^{3}$ To test the effect of a high Pi environment on dentin structure, we next examined overall molar dentin structure and dentin tubules in $k l / k l$ deficient mice, and showed no apparent changes in dentin thickness and dentin tubule number (Figure $4 \mathrm{a}$ and $4 \mathrm{~b}$, left lower panels). Unexpectedly, there were dramatic changes in dentin structure in the compound-deficient root as showed by backscattered SEM and acid etching resin-casted SEM images, in which the dentin was extremely thin with no dentin tubules plus evidence of ectopic calcified tissue formation (Figure $4 \mathrm{a}$ and $4 \mathrm{~b}$, right lower panels). Similar changes were also observed in the incisor structures using H\&E (Figure 5a) and acid etching SEM (Figure 5b). The above data suggest that the loss of the Dmp1 gene or an increase in Pi alone had either minor or no effect on dentin structure. However, striking pathological changes in the dentin structure occurred when both factors were combined together.

$D m p 1^{-/-} / \mathrm{kl} / \mathrm{kl}$ mice display massive apoptosis in ameloblasts and odontoblasts

DMP1 is expressed in both ameloblasts and odontoblasts. ${ }^{24-26}$ To understand why such destructive changes occurred in the compound dentin and enamel, we examined cell apoptosis using the TUNEL assay. As showed in Figure 6a, few TUNEL-positive cells were observed in WT and Dmp1-null odontoblast cells (upper images). In contrast, more positive odontoblasts were shown in the $k l / k l$-deficient odontoblasts (left lower image). In the compound-deficient incisor, few healthy odontoblasts were observed (right lower image). Interestingly, more TUNEL-positive ameloblasts were observed in the $D m p 1^{-/-}$and $k l / k l$-deficient separately (Figure 6b). In the compound-deficient incisoral ameloblasts, almost all cells were positive with strong staining (Figure 6b, right lower image). The above data clearly showed a sharp increase in apoptotic odontoblasts and ameloblasts in the compound-deficient

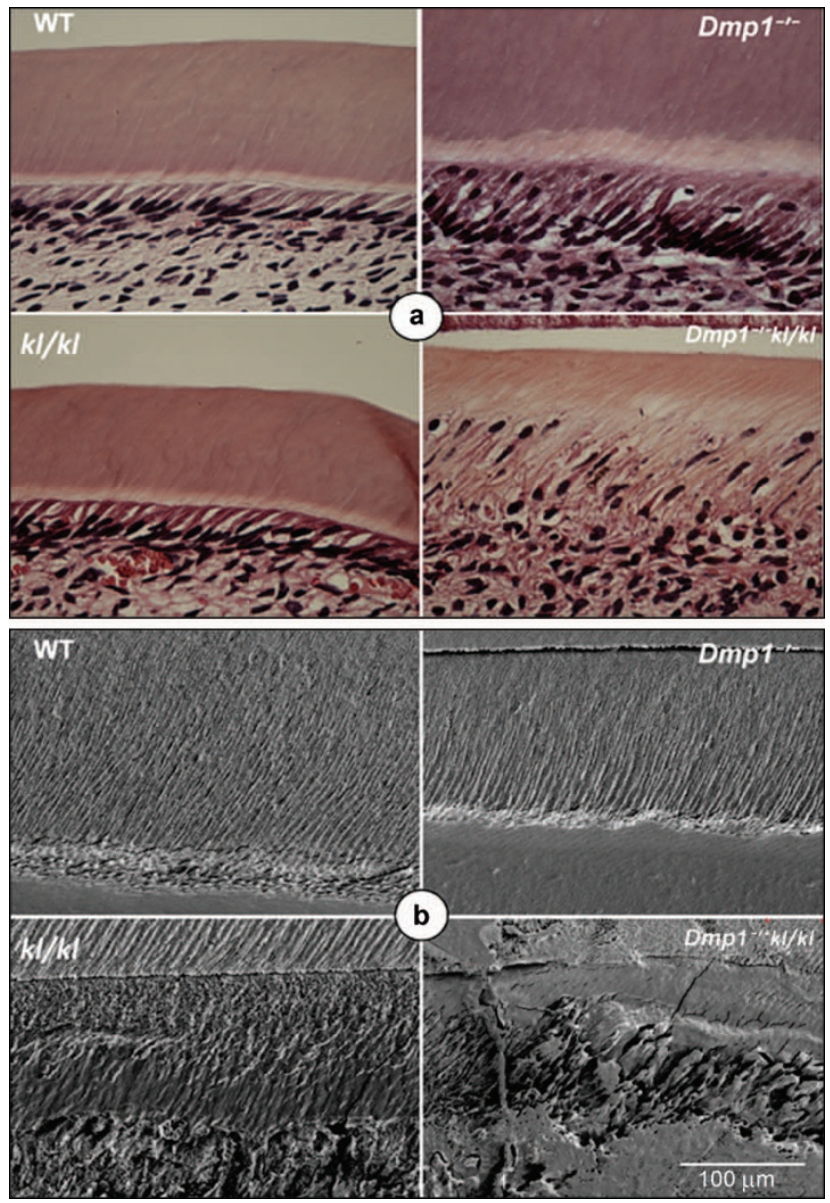

Figure 5 Defective incisor dentin structure of the compound deficient mice. (a) Representative H\&E-stained image revealed an increase in predentin in $D m p 1^{-1-}$ mice (upper right image), little changes in $\mathrm{kl} / \mathrm{kl}$ dentin tubules (lower left image); and few polarized odontoblasts in the compound-deficient pulp; and (b) acid etching resin-casted SEM images revealed no apparent changes in $D m p 1^{-1-}$ dentin tubules except thin dentin thickness (upper right image), abnormal changes in $\mathrm{kl} / \mathrm{kl}$ dentin tubules (lower left image); and a loss of dentin feature in the compound-deficient mice (lower right image). H\&E, hematoxylin and eosin; SEM, scanning electron microscopy. 

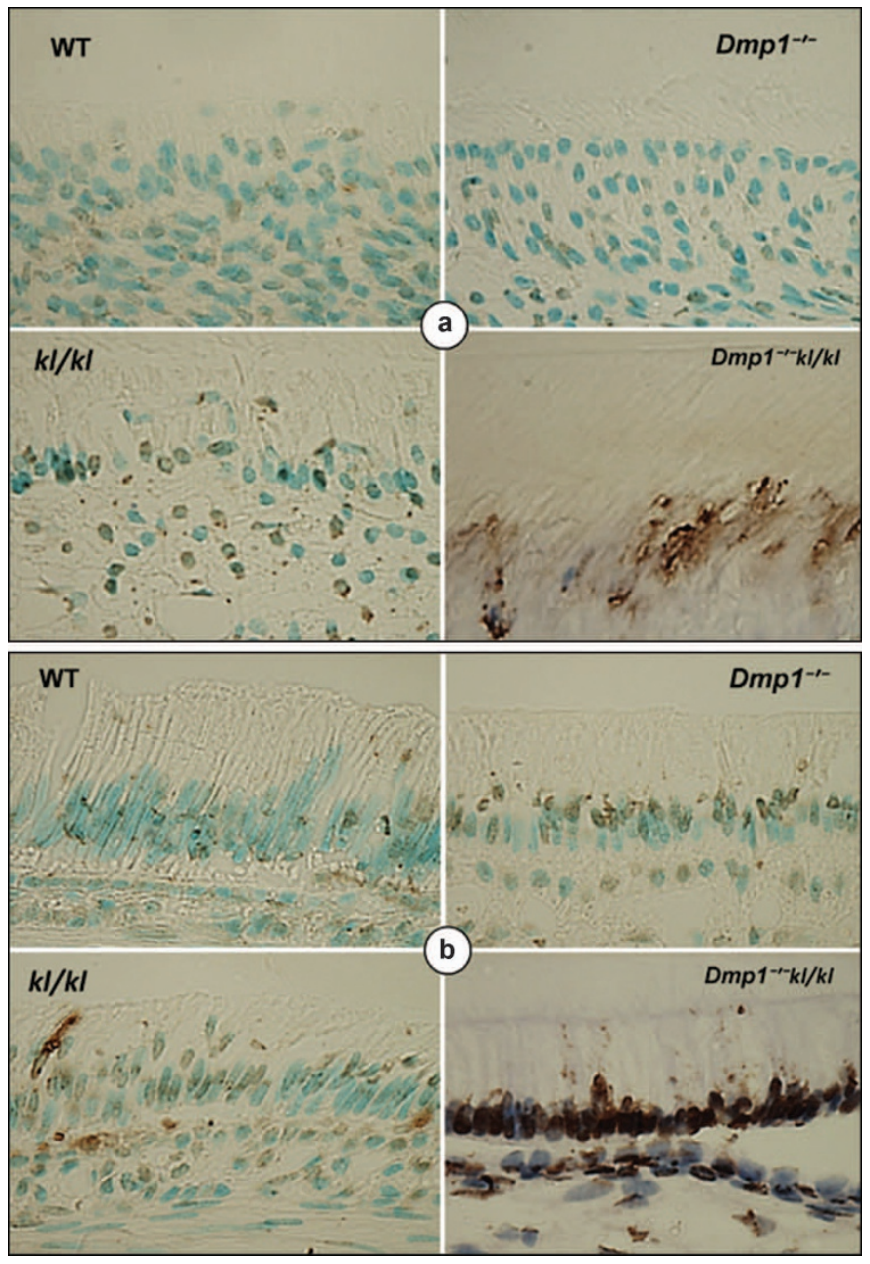

Figure 6 TUNEL staining of cross-section of the incisors in Dmp $1^{-/-}$, Klothodeficient and the compound-deficient odontoblasts and ameloblasts at the age of 6 weeks. (a) TUNEL staining images showed little changes in the Dmp1 incisor odontoblasts (upper right image), a sharp increase of apoptotic odontoblasts in the $\mathrm{kl} / \mathrm{k} / \mathrm{mice}$ (lower left image), and a strong staining signal with no odontoblast cells in the compound deficient mice (lower right image); and (b) TUNEL staining images revealed moderate increase in apoptotic ameloblasts in the $D m p 1^{-/}$ incisor, much more apoptotic ameloblasts in the $\mathrm{kl} / \mathrm{k} / \mathrm{mice}$ (lower left image), and a strong staining signal in almost all ameloblast cells in the compound deficient mice (lower right image).

mice, which is likely a cause for ectopic ossification in a dentin and severe enamel phenotype.

\section{DISCUSSION}

Our previous studies showed that both DMP1 and Pi homeostasis (abnormally high or low) are critical for bone and soft tissues such as blood vessels and kidney. ${ }^{19,27}$ Interestingly, DMP1 is also transiently expressed in developing ameloblasts, ${ }^{18}$ but Dmp1-null mice showed no apparent changes in enamel. Whether DMP1 plays a role in enamel formation or not still remains unclear. In this study, we challenged Dmp1-null mice by crossing them to Klotho-deficient mice, a hyperphosphatemia model. ${ }^{28-29}$ Our findings suggest that: (i) DMP1 has an important local role not only in dentinogenesis, but also in amelogenesis; and (ii) that in the ameloblasts and odontoblasts, DMP1 has a protective role against apoptotic effects of hyperphosphatemia secondary to a Klothodeficient phenotype.
Dmp1-null mice have been reported to develop hypophosphatemia, ${ }^{5,24}$ undergo partial failure of the maturation of predentin into dentin, and pulp cavity and root canal expansion during postnatal tooth development. ${ }^{3} \mathrm{DMP} 1$ has been also shown to have a regulatory effect on Dspp mRNA expression which gives rise to DSP and DPP. ${ }^{30}$ Our recent studies revealed a successful rescue of Dmp1-null dentin phenotype by the targeted expression of the Dspp transgene in the null odontoblasts without $\mathrm{Pi}$ homeostasis changes, supporting a direct role of DMP1 via Dspp which is under control of Dmp1 (Gibson et al., unpubl. data).

Deletion of Dmp1 alone has little effect on enamel formation (Figures 1 and 2). Similarly, there was no apparent change in molar or in the distal incisoral region of the compound-deficient mice. But the proximal enamel region of the compound-deficient mice displays an extremely severe phenotype with little mineralized enamel remaining (Figure la and $1 \mathrm{~b}$ ). The most likely reason for this difference is that enamel in molars and the distal region of the incisor are formed during an embryonic stage, where the serum Pi level in the healthy mother was largely normal. The severe enamel defect in the compound-deficient mice is directly linked to massive ameloblast apoptosis (Figure 6b). There is an increase in apoptosis in both Dmpl-null and $\mathrm{kl} / \mathrm{kl}$-deficient ameloblasts, but we did not observe apparent changes in their enamel, which could be compensated by the abundance of healthy ameloblast cells compared to the number of apoptotic cells. Interestingly, apoptotic events in the ameloblasts did not trigger ectopic ossification, which occurs in the root canal in the compound-deficient mice (see below).

The formation of an ectopic matrix was previously reported in the Klotho and Fgf23-deficient animals. ${ }^{14-15}$ Similarly, a dystrophic pulp calcification, root dilaceration, and a thistle shaped pulp have been reported in the tumoral calcinosis patients who are deficient in FGF23 and have increased phosphate levels. ${ }^{31-32}$ This ectopic ossification is linked to an increase in apoptosis of the odontoblasts. ${ }^{33}$ However, the ectopic ossification and apoptosis in our compound-deficient mice are much more severe than the ossified tissues reported from within the literature, supporting the notion that the antiapoptotic role of DMP1 is critical in hyperphosphatemia. Currently, we do not know why the ectopic ossified tissue appears to be closer to bone or cementum-like tissue in structure. In addition, we do not understand why in the same high Pi environment there is no ectopic ossification in the ameloblast region.

In conclusion, DMP1 has an important protective role in both amelogenesis and dentinogenesis in hyperphosphatemia. Absence of DMP1 results in extremely thin dentin and the formation of an ectopic mineralized matrix within the pulp root canal. It shows that DMP1 has an important local role in the protection of both the odontoblasts and the ameloblasts.

\section{ACKNOWLEDGMENTS}

This study was supported by NIH grants Jian-Quan Feng (DE018486) and to Chun-Lin Qin (DE005092), and State Key Laboratory of Oral Diseases Open Funding (SKLODOF2010-03) to Jian-Quan Feng. Klotho-mutant mice were generously provided by Dr Makoto Kuro-o from University of TX SW Medical Center, Dallas, TX, USA.

1 Fisher LW, Jain A, Tayback $\mathrm{M}$ et al. Small integrin binding ligand $\mathrm{N}$-linked glycoprotein gene family expression in different cancers. Clin Cancer Res 2004; 10(24): 85018511.

2 Qin C, D'Souza R, Feng JQ. Dentin matrix protein 1 (DMP1): new and important roles for biomineralization and phosphate homeostasis. J Dent Res 2007; 86(12): 1134 1141 . 
3 Ye L, MacDougall M, Zhang S et al. Deletion of dentin matrix protein-1 leads to a partial failure of maturation of predentin into dentin, hypomineralization, and expanded cavities of pulp and root canal during postnatal tooth development. J Bio Chem 2004; 279(18): 19141-19148.

4 Lu Y, Ye L, Yu S et al. Rescue of odontogenesis in Dmp1-deficient mice by targeted reexpression of DMP1 reveals roles for DMP1 in early odontogenesis and dentin apposition in vivo. Dev Biol 2007; 303(1): 191-201.

5 Feng JQ, Ward LM, Liu S et al. Loss of DMP1 causes rickets and osteomalacia and identifies a role for osteocytes in mineral metabolism. Nat Genet 2006; 38(11): 1310-1315.

6 Lorenz-Depiereux B, Bastepe M, Benet-Pages A et al. DMP1 mutations in autosoma recessive hypophosphatemia implicate a bone matrix protein in the regulation of phosphate homeostasis. Nat Genet 2006; 38(11): 1248-1250.

7 Jiang B, Cao Z, Lu Y et al. DMP1 C-terminal mutant mice recapture the human ARHR tooth phenotype. J Bone Miner Res 2010; 25(10): 2155-2164.

8 Lu Y, Yuan B, Qin C et al. The biological function of DMP-1 in osteocyte maturation is mediated by its 57-kDa C-terminal fragment. J Bone Miner Res 2011; 26(2): 331-340.

9 Martin A, Quarles LD. Evidence for FGF23 involvement in a bone-kidney axis regulating bone mineralization and systemic phosphate and vitamin $D$ homeostasis. Adv Exp Med Biol 2012; 728: 65-83.

10 Nakatani T, Sarraj B, Ohnishi M et al. In vivo genetic evidence for Klotho-dependent, fibroblast growth factor 23 (Fgf23)-mediated regulation of systemic phosphate homeostasis. FASEB J 2009; 23(2): 433-441.

11 Kurosu H, Ogawa $\mathrm{Y}$, Miyoshi M et al. Regulation of fibroblast growth factor-23 signaling by Klotho. J Biol Chem 2006; 281(10): 6120-6123.

12 Li SA, Watanabe M, Yamada $\mathrm{H}$ et al. Immunohistochemical localization of Klotho protein in brain, kidney, and reproductive organs of mice. Cell Struct Funct 2004; 29(4): 91-99.

13 Urakawa I, Yamazaki Y, Shimada T et al. Klotho converts canonical FGF receptor into a specific receptor for FGF23. Nature 2006; 444(7120): 770-774.

14 Suzuki H, Amizuka N, Oda K et al. Involvement of the Klotho protein in dentin formation and mineralization. Anat Rec (Hoboken) 2008; 291(2): 183-190.

15 Chu EY, Fong $\mathrm{H}$, Blethen FA et al. Ablation of systemic phosphate-regulating gene fibroblast growth factor 23 (Fgf23) compromises the dentoalveolar complex. Anat Rec (Hoboken) 2010; 293(7): 1214-1226.

16 Bai XY, Miao D, Goltzman D et al. The autosomal dominant hypophosphatemic rickets $\mathrm{R} 176 \mathrm{Q}$ mutation in fibroblast growth factor 23 resists proteolytic cleavage and enhances in vivo biological potency. J Biol Chem 2003; 278(11): 9843-9849.

17 White KE, Carn G, Lorenz-Depiereux B et al. Autosomal-dominant hypophosphatemic rickets (ADHR) mutations stabilize FGF-23. Kidney Int 2001; 60(6): 2079-2086.

18 Martinez EF, da Silva LA, Furuse C et al. Dentin matrix protein 1 (DMP1) expression in developing human teeth. Braz Dent J 2009; 20(5): 365-369.
19 Rangiani A, Cao Z, Sun Y et al. Protective roles of DMP1 in high phosphate homeostasis. PLoS One 2012; 7(8): e42329.

20 Kuro-o M, Matsumura Y, Aizawa $\mathrm{H}$ et al. Mutation of the mouse Klotho gene leads to a syndrome resembling ageing. Nature 1997; 390(6655): 45-51.

21 Feng JQ, Huang H, Lu Y et al. The Dentin matrix protein 1 (Dmp1) is specifically expressed in mineralized, but not soft, tissues during development. J Dent Res 2003; 82(10): 776-780.

22 Tsujikawa H, Kurotaki Y, Fujimori T et al. Klotho, a gene related to a syndrome resembling human premature aging, functions in a negative regulatory circuit of vitamin D endocrine system. Mol Endocrinol 2003; 17(12): 2393-2403.

23 Brownstein CA, Zhang J, Stillman A et al. Increased bone volume and correction of HYP mouse hypophosphatemia in the Klotho/HYP mouse. Endocrinology 2010; 151(2): 492-501.

24 Ye L, Mishina Y, Chen D et al. Dmp1-deficient mice display severe defects in cartilage formation responsible for a chondrodysplasia-like phenotype. J Biol Chem 2005; 280(7): 6197-6203.

25 Ling Y, Rios HF, Myers ER et al. DMP1 depletion decreases bone mineralization in vivo: an FTIR imaging analysis. J Bone Miner Res 2005; 20(12): 2169-2177.

26 Liu H, Fergusson MM, Castilho RM et al. Augmented Wnt signaling in a mammalian model of accelerated aging. Science 2007; 317(5839): 803-806.

27 Lv K, Huang H, LuY et al. Circling behavior developed in Dmp1 null mice is due to bone defects in the vestibular apparatus. Int J Biol Sci 2010; 6(6): 537-545.

28 Kuro-o M. Klotho and aging. Biochim Biophys Acta 2009; 1790(10): 1049-1058.

29 Kuro-o M. Klotho as a regulator of fibroblast growth factor signaling and phosphate/ calcium metabolism. Curr Opin Nephrol Hypertens 2006; 15(4): 437-441.

30 Narayanan K, Gajjeraman S, Ramachandran A et al. Dentin matrix protein 1 regulates dentin sialophosphoprotein gene transcription during early odontoblast differentiation. J Biol Chem 2006; 281(28): 19064-19071.

31 Yoshimura I, Suzuki S, Hayakawa M. Application of Cre-loxP system to the urinary tract and cancer gene therapy. Mol Urol 2001; 5(2): 81-84.

32 Naikmasur V, Guttal K, Bhargava P et al. Tumoral calcinosis with dental manifestationsa case report. Dent Update 2008; 35(2): 134-136, 138.

33 Suzuki $\mathrm{H}$, Amizuka N, Oda K et al. Histological evidence of the altered distribution of osteocytes and bone matrix synthesis in Klotho-deficient mice. Arch Histol Cytol 2005; 68(5): 371-381.

(c) This work is licensed under a Creative Commons

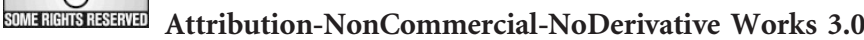
Unported License. To view a copy of this license, visit http:// creativecommons.org/licenses/by-nc-nd/3.0 